\title{
Urticarial vasculitis as an initial manifestation of colonic carcinoma: a case report and review of the literature
}

\author{
A.A. Younis \\ Rheumatology Department, Faruk Medical City, Iraq
}

\begin{abstract}
SUMMARY
Cutaneous vasculitis may behave as a paraneoplastic syndrome. Paraneoplastic vasculitis as a phenomenon of colonic adenocarcinoma has been described in the literature. To the best of my knowledge, this is the first case report of urticarial vasculitis being an initial presentation of signet-ring cell carcinoma of the colon. I here report a case of 27-year-old woman who had rash, arthritis, and episodic bleeding per rectum. The rash was biopsy-proven to be leukocytoclastic vasculitis. There was only partial response to high-dose steroid, and rectal bleeding continued. Colonoscopy revealed a stenosing growth of sigmoid colon, which proved to be signet ring cell carcinoma of the colon. It is important to remain vigilant with regard to the association between leukocytoclastic vasculitis and malignancies.
\end{abstract}

Key words: Leukocytoclastic vasculitis; Urticarial vasculitis; Colonic carcinoma; Paraneoplastic.

Reumatismo, 2018; 70 (4): 259-263

\section{INTRODUCTION}

$\mathrm{T}$ he term cutaneous vasculitis includes a broad and heterogeneous spectrum of syndromes clinically characterized by predominant skin involvement, with histopathologic findings of vascular inflammation and blood vessel damage (1). Although isolated cutaneous vasculitis is usually a benign process, in some cases it may be associated with malignancy and may behave as a paraneoplastic syndrome (2). Cutaneous leukocytoclastic vasculitides are the most frequent paraneoplastic types, and their clinical manifestations usually appear before or concurrent with the diagnosis or relapse of a tumor (3). Urticarial vasculitis (UV) is considered a clinicopathologic entity characterized by cutaneous manifestations of urticaria along with histopathologic findings of leukocytoclastic vasculitis of the small vessels (4). Although UV is most commonly idiopathic, it can occur in association with autoimmune diseases, drug reactions, infections, or malignancy (5). Urticarial vasculitis has been associated with multiple myeloma, non-Hodgkin lymphoma, metastatic teratoma from a testicular tumor, metastatic adenocarcinoma of the colon, and renal carcinoma (6). Paraneoplastic leukocytoclastic vasculitis as a phenomenon of colonic cancer has been described in the literature (7). To the best of my knowledge, this is the first case report of urticarial vasculitis being an initial presentation of signet-ring cell carcinoma of the colon.

\section{CASE REPORT}

A 27-year-old woman presented to the rheumatology outpatient clinic of Faruk medical city with a 10-day history of multiple-joint pain and skin rash. The patient was not known to have any chronic illness, and had had uncomplicated vaginal delivery of her first baby 2 months earlier. She was well until 10 days prior to presentation, when she developed recurrent, intensely pruritic, and painful, burning skin rash involving the face, trunk, upper and lower extremities. The lesions persisted for 24-48 $\mathrm{h}$ and then subsided, sometimes leaving residual hyperpigmentation. $\overline{\text { Corresponding author: }}$ Ali Abdulrahman Younis Rheumatology Department, Faruk Medical City, Malik Mahmood Circle Street, Sulaimani, Kurdistan, Iraq. E-mail: ali.younis7622@yahoo.com 
In addition, the patient admitted having multiple-joint pain symmetrically involving small and large joints in the upper and lower limbs. The pain was continuous, worse in the morning, partially relieved by simple activities, associated with morning stiffness for more than $30 \mathrm{~min}$, and joint swelling involving mainly the wrists, ankles and small joints of the hands and feet. The patient also suffered from low-grade

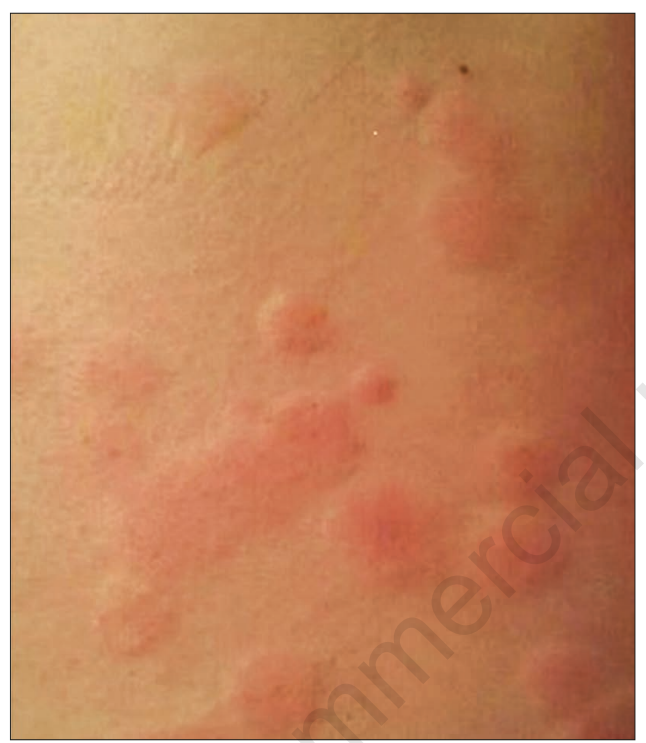

Figure 1 - Skin examination revealing urticarial plaques in variable sizes throughout patient's body.

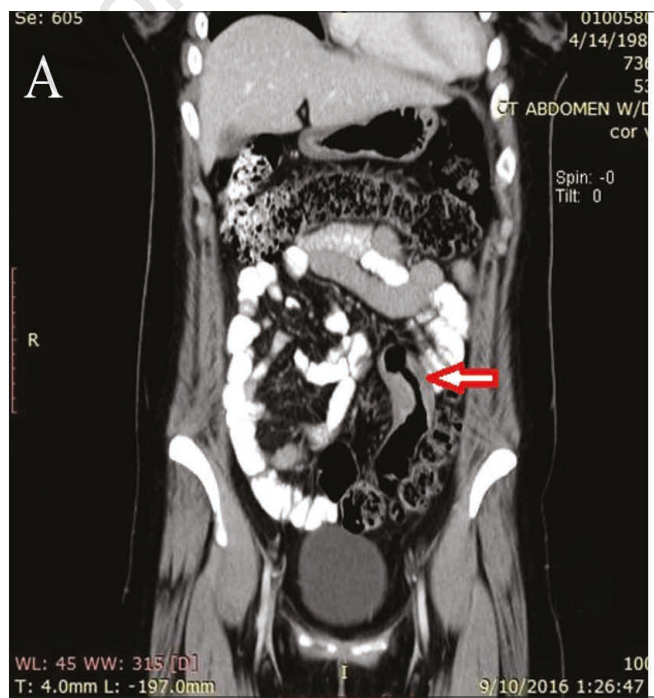

fever, lip swelling, shortness of breath, headache, nausea, diffuse abdominal pain, frequent loose motions with occasional bleeding per rectum. She denied having photosensitivity, eye dryness, mouth ulcerations, excessive hair loss, chest pain, Raynaud's phenomena, change in urine color, and weight loss. On further questioning there were no obvious triggers such as recent drug ingestion or past history of any allergies. Family history was noncontributory. The patient consulted a dermatologist and a gastroenterologist, and she was diagnosed with urticaria secondary to gastrointestinal infection. There was a poor response to the prescribed treatment, including antihistamine and antibiotics.

On examination the patient was conscious, looked ill, tired, with pale complexion. Examination for vital signs revealed blood pressure of 120/70 mm Hg with no postural drop, pulse of $95 \mathrm{bpm}$, respiratory rate of 18 breaths/min and temperature of $37.8^{\circ} \mathrm{C}$. Skin examination revealed urticarial plaques in variable sizes throughout her body, which were tender to palpation (Figure 1). Cardiac, respiratory, and neurological examinations were within the norm. Abdominal examination revealed tenderness in suprapubic region and left iliac fossa with positive bowel sounds. There was bilateral proximal interphalangeal, meta-

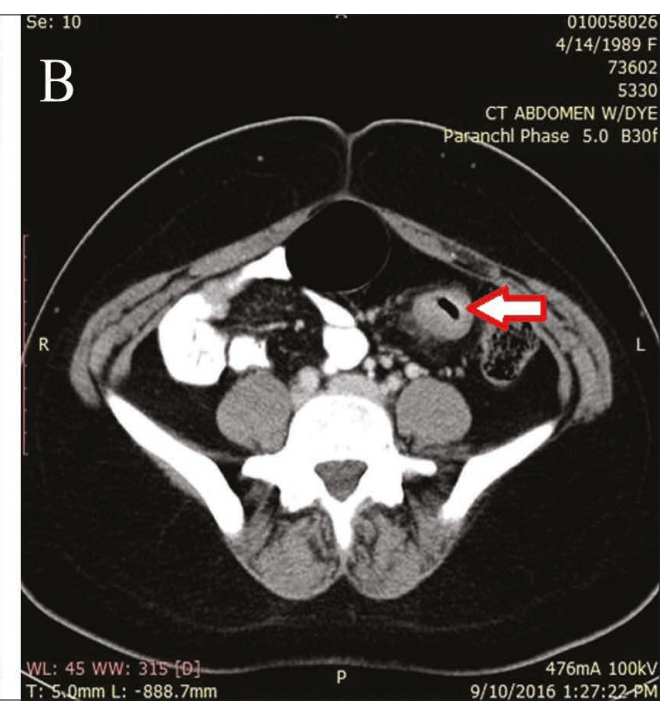

Figure 2 - A-B) Computed tomography scan of the abdomen revealing a short segment of irregular circumferential mural wall thickening in the sigmoid colon. 
carpophalangeal, wrist, elbow, ankle, and knee joint arthritis. Deltoid and quadriceps muscles were tender to palpation.

Laboratory testing including complete blood count, liver, kidney and thyroid function tests, inflammatory markers, antinuclear antibody (ANA), extractable nuclear antigen (ENA) tests, double-stranded DNA, rheumatoid factor, c-ANCA, p-AN$\mathrm{CA}$, serum immunoglobulins, $\mathrm{HBsAg}$, anti-HCV antibodies, and complements were all within normal limits, along with normal urinalysis, echocardiography, chest X-ray, and abdominal ultrasound. Skin biopsy revealed leukocytoclastic vasculitis. A diagnosis of urticarial vasculitis, possibly complicated by mesenteric ischemia was done. The patient was commenced on methylprednisolone vial $1 \mathrm{gm}$ intravenous infusion for 3 days followed by prednisolone tab $40 \mathrm{mg}$ daily in 2 divided doses, with fexofenadine $180 \mathrm{mg}$ tab once daily, and azathioprine $50 \mathrm{mg}$ twice daily. Initially the patient's rash and to a lesser extent her joint symptoms and abdominal pain partially improved, but the rectal bleeding continued to occur episodically. On reviewing the patient three weeks later, she was still having abdominal pain and rectal bleeding, in addition to rash and joint pain.

A computed tomography (CT) scan of the abdomen with oral and intravenous contrast revealed a short segment of irregular circumferential mural wall thickening in the sigmoid colon, moderately encroaching upon the lumen (Figure 2). The abdominal aorta and its branches were normal. A provisional diagnosis of colonic carcinoma was made with a differential diagnosis of ischemic contracture. A gastroenterologist's opinion was therefore obtained with a view to having a colonoscopy.

The patient underwent total colonoscopy, which revealed a stenosing growth with ulcerated friable mucosa in the sigmoid colon (Figure 3). A biopsy was taken which revealed atypical cells with intracellular mucin, in keeping with signet ring cell carcinoma (Figure 4). The diagnosis was confirmed with an immunohistochemical study. The patient was operated in India; a radical sigmoidectomy with rectal resec-
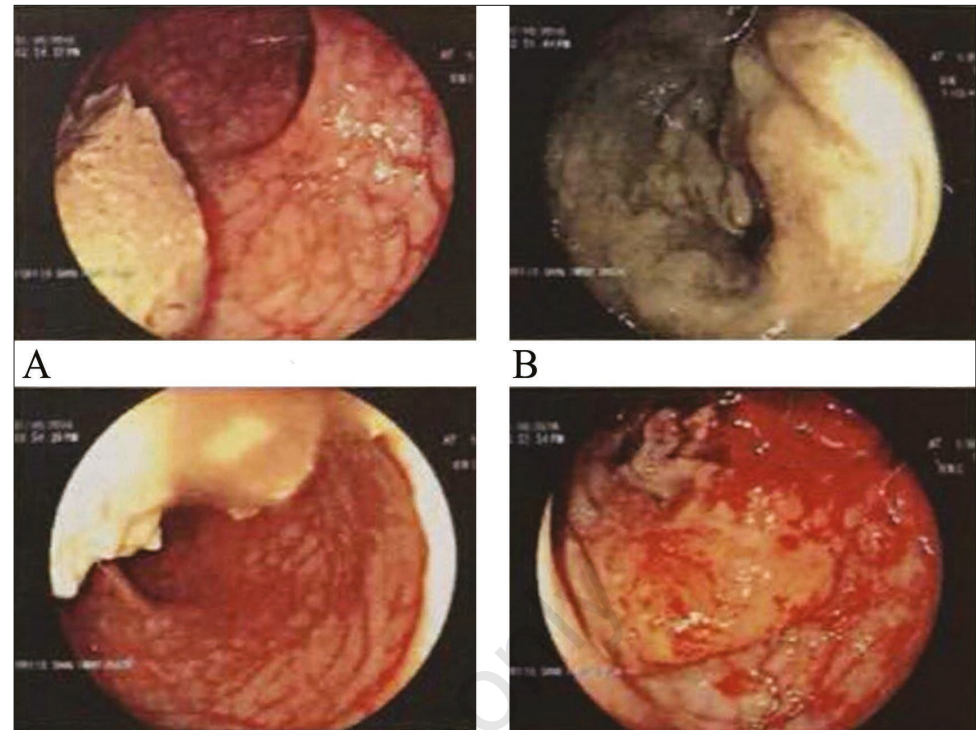

B

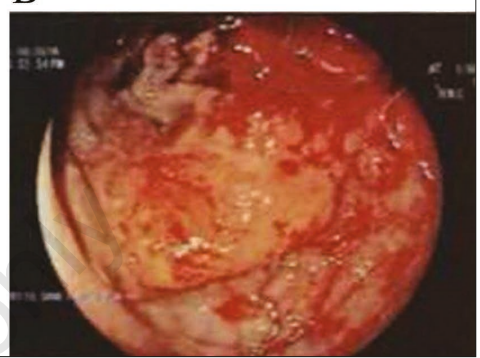

Figure 3 - A-B) Patient's colonoscopy revealing a stenosing growth with ulcerated friable mucosa in the sigmoid colon.

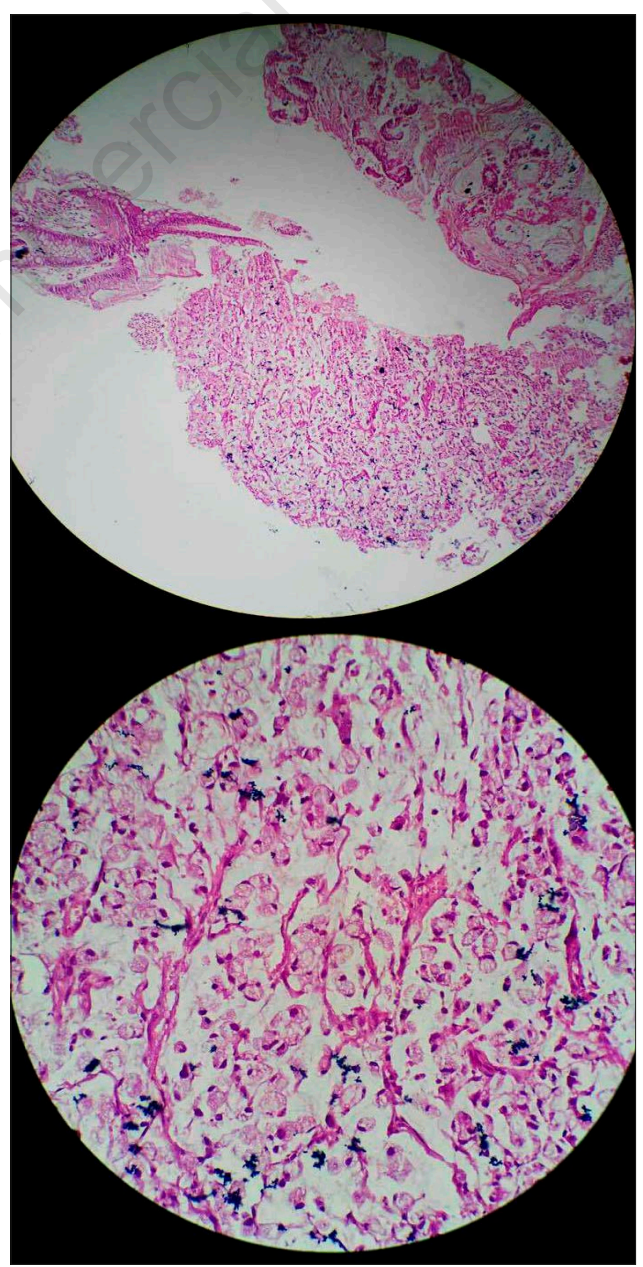

Figure 4 - A-B) Biopsy revealing atypical cells with intracellular mucin with signet ring cell carcinoma. 
tion, colorectal anastomosis with covering loop ileostomy was performed, followed by chemotherapy. A post-operation 18-fluorodeoxyglucose positron emission tomography CT $\left({ }^{18} \mathrm{FDG}\right.$ PET/CT) scan showed no evidence of metastasis. The patient's rash and arthritis completely resolved following surgery, despite being off treatment, and on follow-up 6 months later, the patient was still free from symptoms. Accordingly, the patient most likely had paraneoplastic urticarial vasculitis complicating signet ring carcinoma of the colon.

\section{DISCUSSION}

Cutaneous vasculitis may behave as a paraneoplastic syndrome (2). The available data on paraneoplastic vasculitis has been generally retrieved from relatively small series or from case reports based on a few patients (1). Vasculitis was reported to occur during the course of malignancies in $2.3-8 \%$ of the patients (8). The frequency of vasculitis in patients with cancer was estimated to be at 1 in 1800 for hematological malignancies and 1 in 80,800 for solid tumors (9). Almost $80 \%$ of patients have an associated hematological malignancy, while the remaining $20 \%$ or so have a solid tumor or a tumor of unknown origin (6). In decreasing order, the most common solid malignancies described in association with vasculitis are lung, colon adenocarcinoma, renal carcinoma, cancer of the urinary bladder, and prostate and breast cancer (10). The paraneoplastic vasculitides may be of small, medium, and large-sized vessels, but small vessel vasculitis is the most frequently observed (7). The most common site for paraneoplastic vasculitis is the skin, and around $50 \%-60 \%$ of paraneoplastic cutaneous vasculitis is leukocytoclastic vasculitis, and $15 \%$ is Schönlein-Henoch purpura (9); however, urticarial vasculitis has been described as a rare association with visceral and hematological malignancy (11).

There are several hypotheses regarding the pathogenesis of paraneoplastic vasculitis, including the formation of antigen-antibody immune complexes associated with the tumor, direct vascular damage to en- dothelial cells due to a cross reaction with antigens in the tumor cells, the release of various cytokines, causing endothelial injury, and the induction of a delayed hypersensitivity reaction by deposition of cancer proteins on vessel walls (7).

Cutaneous vasculitis may predate the discovery of the malignancy, coincide with it, occur after malignancy recognition, or herald malignancy recurrence (7). In most cases, paraneoplastic vasculitis antedates the diagnosis of malignancy (1).

In order to be considered as paraneoplastic vasculitis, it should have a parallel course to the underlying tumor and reappear in case of recurrence (12). A paraneoplastic origin is suggested by the temporal relationship between both conditions; vasculitis appearance varies from 25 months proceeding to nine months following cancer diagnosis (13). The relationship is further supported by the concordance of disease activity and treatment response for both cancer and vasculitis $(7,14)$. In the described case, the presentation of both cancer and vasculitis was nearly at the same time and diagnosis of both conditions was within 2 months. This suggests that the 2 processes were related and not chance occurrences. The relationship is also supported by the lack of response of vasculitis to glucocorticoid therapy prior to the initiation of treatment of malignancy, and the resolution of vasculitis concurrently with the specific treatment of malignancy.

The clinical manifestations of paraneoplastic vasculitides are similar to those seen in patients with primary vasculitis (12). Screening all patients with cutaneous vasculitis for a malignancy would not be cost effective or justified. However, clinical evaluation including history, physical examination, and monitoring the clinical course of the disease is strongly recommended. Additional testing including imaging studies may be warranted based on initial clinical evaluation and laboratory findings. Age-appropriate cancer screening should be ensured. Malignancy should be suspected when cutaneous vasculitis does not respond appropriately to corticosteroids or when it is refractory to treatment. It should also be suspected in the 
presence of atypical clinical features such as profound weight loss or other constitutional symptoms (15).

Treatment and prognosis of paraneoplastic vasculitis is generally related to the underlying neoplasm. In some cases, treatment with glucocorticoids may be required, either alone or in combination with immunosuppressive agents (16). However, paraneoplastic vasculitis often has poor response to conventional therapy directed against the vasculitis itself. In one series of 13 patients with cutaneous vasculitis and lymphoproliferative or myeloproliferative disorders, the symptoms of vasculitis responded poorly to therapy with non-steroidal antiinflammatory drugs, glucocorticoids, and antihistamines (17). Likewise, Hutson and Hoffman noticed a general concurrence between improvement in vasculitis symptoms and definitive treatment for the associated underlying malignancy (14). In a more recent study of cutaneous vasculitis associated with solid tumors (most commonly lung, breast, and prostate), investigators found that vasculitis responded significantly to immunosuppressive therapy, although concurrent treatments for the underlying malignancy were undertaken (18).

\section{CONCLUSIONS}

It is important to remain vigilant with regard to the association between leukocytoclastic vasculitis and malignancies. A diagnosis of vasculitis requires a search for malignancies as well as other possible etiologies. This is particularly of relevance when vasculitis has a poor response to conventional treatment, as well as when there is an unclear correlation with previous infections or systemic autoimmune diseases.

Conflict of interest: the author declares no conflict of interest.

\section{REFERENCES}

1. Loricera J, Calvo-Rio V, Ortiz-Sanjuan F, et al. The spectrum of paraneoplastic cutaneous vasculitis in a defined population: incidence and clinical features. Medicine (Baltimore). 2013; 92: 331-43.
2. Buggiani G, Krysenka A, Grazzini M, et al. Paraneoplastic vasculitis and paraneoplastic vascular syndromes. Dermatol Ther. 2010; 23 : 597-605.

3. Racanelli V, Prete M, Minoia C, Favoino E, Perosa F. Rheumatic disorders as paraneoplastic syndromes. Autoimmun Rev. 2008; 7: 352-8.

4. Sanchez NP, Winkelmann RK, Schroeter AL, Dicken $\mathrm{CH}$. The clinical and histopathological spectrums of urticarial vasculitis: study of forty cases. J Am Acad Dermatol. 1982; 7: 599 Y605.

5. Wisnieski JJ. Urticarial vasculitis. Curr Opin Rheumatol. 2000; 12: 24.

6. Yuste-Chaves M, Unamuno-Pérez P. Cutaneous alerts in systemic malignancy: part I. Actas Dermosifiliogr. 2013; 104: 285-98.

7. Solans-Laque R, Bosch-Gil JA, Perez-Bocanegra $\mathrm{C}$, et al. Paraneoplastic vasculitis in patients with solid tumors: report of 15 cases. J Rheumatol. 2008; 35: 294-304.

8. Fain O, Hamidou M, Cacoub P, et al. Vasculitides associated with malignancies: Analysis of sixty patients. Arthritis Rheum. 2007; 57: 1473-80.

9. Park HJ, Ranganathan P. Neoplastic and paraneoplastic vasculitis, vasculopathy, and hypercoagulability. Rheum Dis Clinics N Am. 2011; 37: 593-606.

10. Arvinius C, González-Pérez A, García-Coiradas J, et al. Paraneoplastic vasculitis associated to pelvic chondrosarcoma: a case report. SICOT-J. 2016; 2: 8.

11. Wilson D, McCluggage WG, Wright GD. Urticarial vasculitis: a paraneoplastic presentation of B-cell non-Hodgkin's lymphoma. Rheumatology (Oxford). 2002; 41: 476-7.

12. Hommel C, Rihova Z, Mokaddem F, Libotte B. pANCA-vasculitis associated with rectal adenocarcinoma. Acta Clin Belg. 2014; 69: 463-6.

13. Diez-Porres L, Rios-Blanco JJ, RoblesMarhuenda A, et al. ANCA-associated vasculitis as paraneoplastic syndrome withcolon cancer: a case report. Lupus. 2005; 14: 632-4.

14. Hutson TE, Hoffman GS. Temporal concurrence of vasculitis and cancer: a report of 12 cases. Arthritis Care Res. 2000; 13: 417-23.

15. Kermani TA, Warrington KJ, Amin S. Malignancy risk in vasculitis. Ther Adv Musculoskel Dis. 2011; 3: 55-63.

16. Kurzrock R, Cohen PR, Markowitz A. Clinical manifestations of vasculitis in patients with solid tumors. Arch Intern Med. 1994; 154: 334-40.

17. Greer JM, Longley S, Edwards NL, et al. Vasculitis associated with malignancy: experience with 13 patients and literature review. Medicine (Baltimore). 1988; 67: 220-30.

18. Podjasek JO, Wetter DA, Pittelkow MR, et al. Cutaneous smallvessel vasculitis associated with solid organ malignancies: the Mayo Clinic experience, 19962009. J Am Acad Dermatol. 2012; 66: e55-65. 